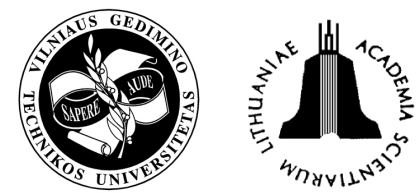

\title{
OPTIMIZING MAINTENANCE RELIABILITY PROGRAM FOR SMALL FLEETS
}

\author{
Željko Marušić ${ }^{1}$, Borivoj Galović ${ }^{2}$, Omer Pita ${ }^{3}$ \\ ${ }^{1,2}$ Faculty of Traffic and Transport Engineering, Dept of Aeronautics, University of Zagreb \\ Vukelićeva 4, 10000 Zagreb, Croatia \\ ${ }^{3}$ Croatia Airlines, POB. 5; 10150 Airport Zagreb, Croatia \\ E-mails: ${ }^{1}$ zeljko.marusic@fpz.hr; ${ }^{2}$ borivoj.galovic@fpz.hr; ${ }^{3}$ omer.pita@croatiaairlines.hr
}

Received 9 January 2007; accepted 2 May 2007

\begin{abstract}
Reliability program is considered to be a very valuable means of achieving better operational performance (through decreased maintenance related problems in operation) and increased flight safety. For this reason, reliability programs are mandated by the regulations for all commercial operators. Depending on the size of the operator, implementation of reliability program can be carried out in various organizational forms. Small fleets represent too small a statistical sample to collect enough information for obtaining statistically significant and accurate data. Therefore usability of reliability program in very small fleets is questionable. The aim of this work is to highlight some problems related to maintenance reliability program for small fleets.
\end{abstract}

Keywords: reliability oriented maintenance, aircraft maintenance, fleet.

\section{Introduction}

Maintenance reliability program is a system of statistical monitoring and reporting of the events related to technical status of an aircraft type during its operation in the operator's fleet. Having in mind that operators operate their fleets in different operational conditions, reliability program has to be carried out for each operator separately to reflect all the differences in the operation that exist between operators. The primary purpose of reliability program is to provide statistical information which could be used for adapting and improving the operator's aircraft maintenance program for a specific fleet of single aircraft type. Using primarily statistical methods, the number of samples and events has to be large enough to obtain trustworthy statistical results [1-4].

Also, small fleets are often monitored by small engineering organizations where engineering manpower is very limited. This leads to very basic and shallow conduct of maintenance reliability program not reaching all aspects of problems in the fleet.

\section{Establishing maintenance reliability program}

To have full benefit, size of the fleet covered by reliability program should be statistically significant. A small number of aircraft (one to five), yielding a small number of technical events, generates statistical information that is not realistic and trustworthy. Comparing Fig 1 and Fig 2 it is visible that smaller fleet generates larger dissipation of data, in this particular case, through sequence of monthly technical dispatch rate data.

In order to receive more realistic results, data from Fig 1 should be smoothened. Theoretically, smoothening of reliability data could be done by:

- calculation of accrued values;

- merging reliability data with that from other operators with similar operation.

Calculation of accrued values is based on calculating events from last three (or more) months of operation on monthly basis for a monthly reliability analysis. This method artificially increases fleet size three (or more) times, consequently smoothening the results three (or more) times. Obtained statistical data still reflects only the observed operator's fleet data without influence from other fleets. However, during performance of analysis of such data, special care should be taken when interpreting one single problem that is repeatedly appearing on one aircraft. Very frequently this occurs because of the nature of a defect (e. g. failure appears occasionally during the flight and can not be reproduced while testing on ground) which hinders troubleshooting efforts and same system error gets fixed and reappears several times before it gets permanently fixed. This data would appear on such accrued report as a fleet problem, which is not the case. So in monthly reliability report all such isolated problems have to be explained and separated from actual ones that are present fleet-wide. 


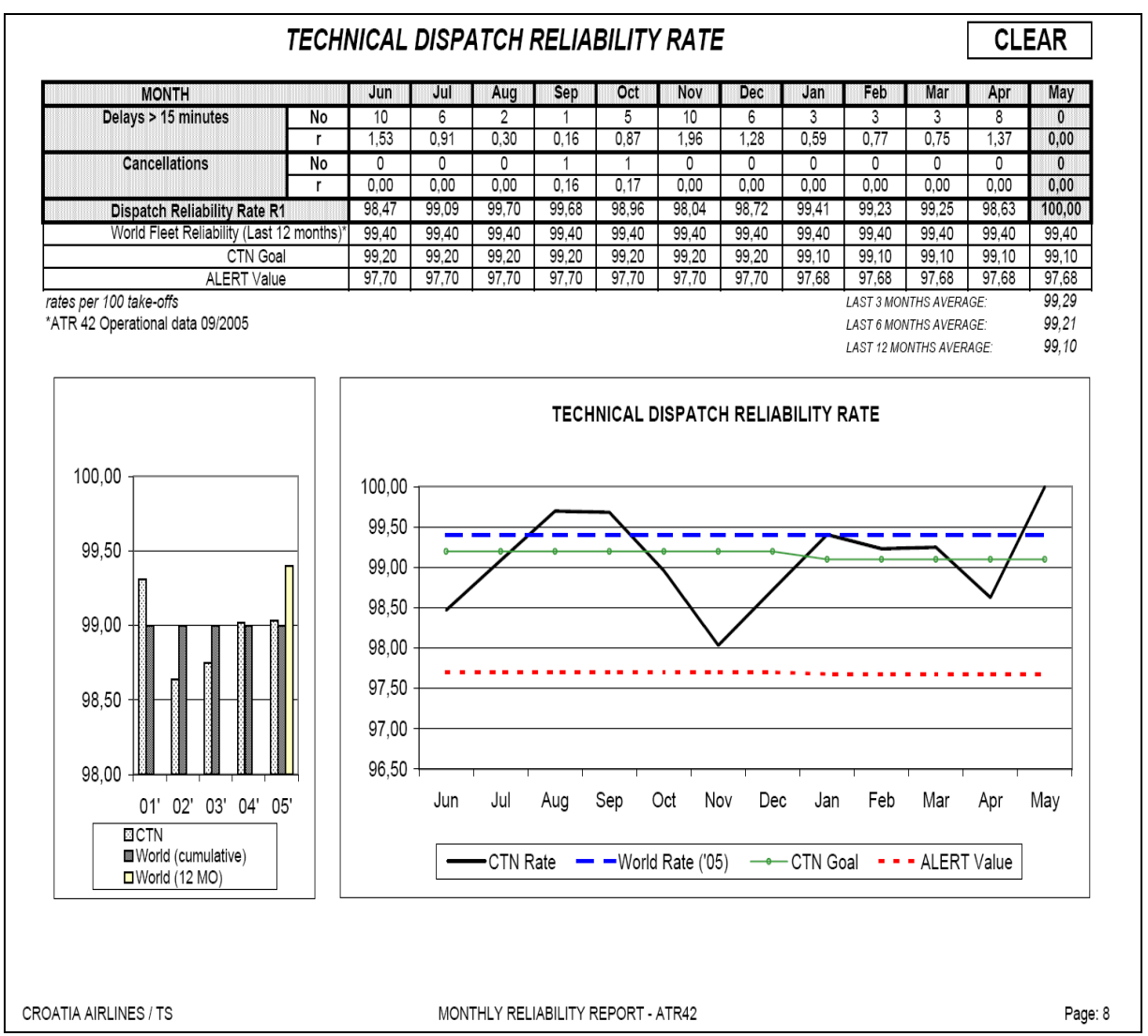

Fig 1. Technical dispatch rate for small fleet of three aircraft ATR42, source: Reliability Report 06/2006; Croatia Airlines

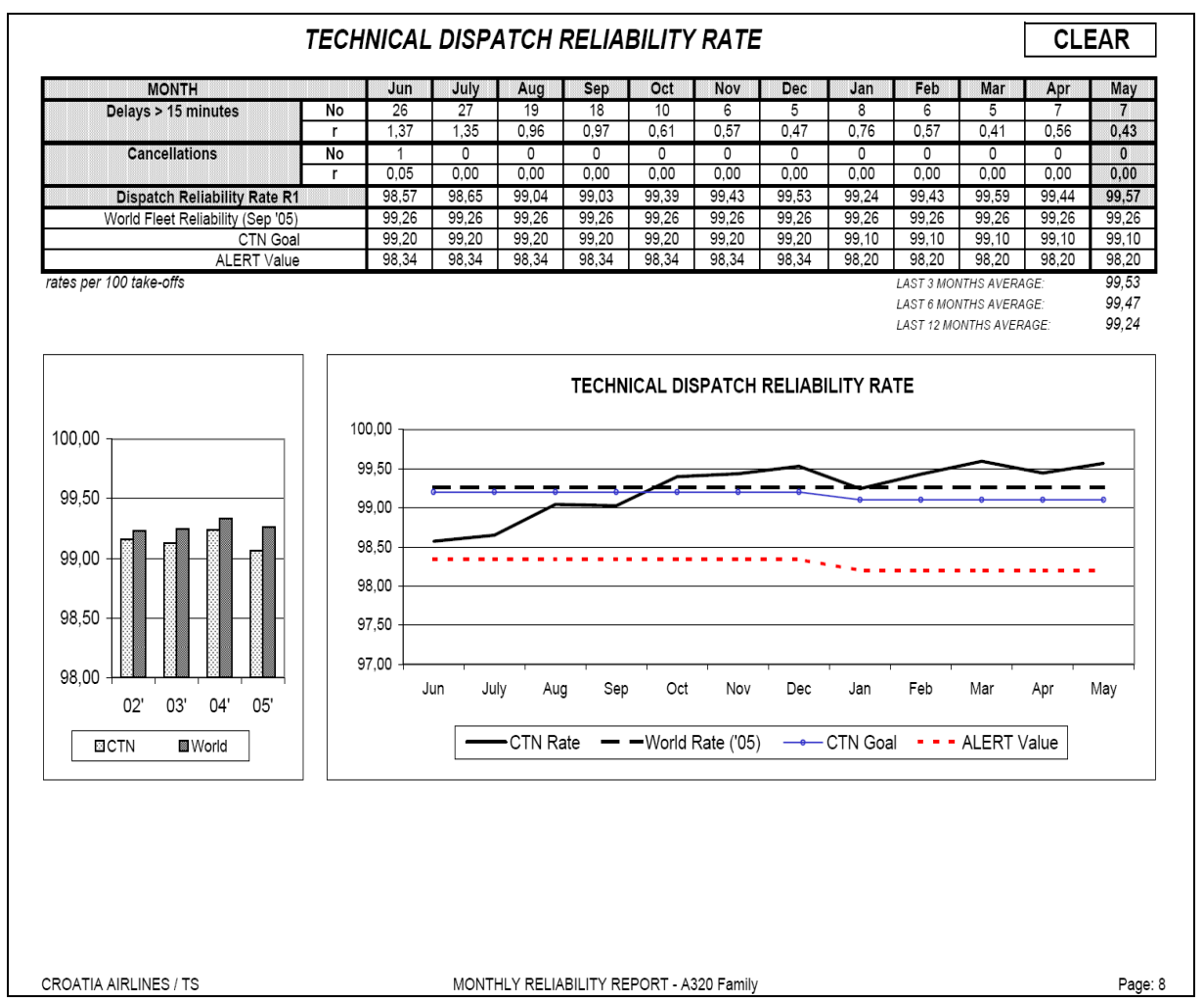

Fig 2. Technical dispatch rate for fleet of eight aircraft A320; source: Reliability report 06/2006, Croatia Airlines 
Another issue that has to be observed, while defining number of months to include in monthly calculation, would be seasonality of operation which at some operators could mean two or more totally different types of operation. Problematic would be the mix of months before and after shift of seasons took place.

Merging more small fleets of similar operators gives synthetic, statistically significant information which applies to different operators participating in the combined program. Drawback of this method is that local deviations of a single operator are spread on all operators participating in the combined reliability program which may lead to wrong conclusions.

Any reliability program that involves method of data smoothening is subject to Authority approval.

\section{Flow of reliability program}

Reliability program is based on fact that inherent reliability level of specific system or component or whole aircraft is changed subject to:

- influence of environment in which the operation is carried out;

- type of operation.

Negative deviation relative to world average reliability of certain aircraft type can be the consequence of specific operation. Therefore, it is important to have reliability program in place to optimize the maintenance program in order to minimize this deviation.

\section{Deployment of reliability program within operator's organization}

Reliability program must be a written program approved by the Authority. It has to specify responsibilities and procedures within the operator's organization, which will ensure successful functioning of reliability program. All basic steps as defined in Fig 3 have to be defined within organizational procedures.

\section{Parameters defined in reliability program}

Operator can vary the number of parameters which are analyzed in reliability program, while basic standard parameters are:

- number of pilots' complaints per 100 flights;

- number of technical delays above $15 \mathrm{~min}$ and cancellations per 1000 flights;

- number of component replacements per 1000 component flight hours;

- number of unscheduled component replacements per 1000 component flight hours;

- number of engine in-flight shut downs per 1000 engine hours;

- number of unscheduled engine changes per 1000 engine hours;

- repetitive pilots' complaints;

- long-lasting technical problems with defect rectification (service difficulty reports);

- significant findings during scheduled base maintenance events.

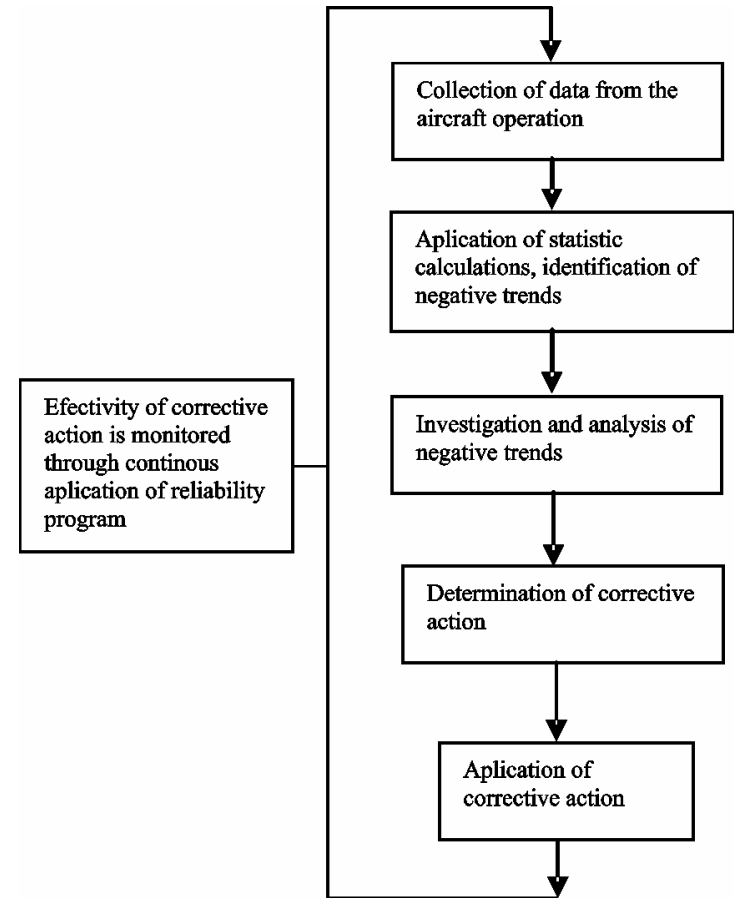

Fig 3. Generic flowchart of reliability program

\subsection{Participation of airworthiness authorities}

In order to monitor its operators, airworthiness authorities have to receive copies of Reliability Control Board meeting minutes and Monthly reliability reports.

Airworthiness authorities have to be informed in advance about date, time and place of scheduled Reliability Control Board meeting, at which Authorities can decide to participate at their own discretion. It is not unusual that members of Airworthiness Authority participate in RCB meetings on regular basis.

Members of Airworthiness Authorities have access to all relevant information related to ongoing Reliability Program.

\subsection{Upper control limits (alert values)}

For every measured parameter, Upper control limit or alert value has to be determined. Alert value is used to recognize and react to significant deviations from statistically acceptable limits in reliability.

Upper control level or alert value is statistical value which shows the limit below which deviations are considered statistically acceptable.

In case of exceeded alert limit value, system reliability is considered to be unstable.

Repeated exceeding of the alert value represents a negative trend which has to be stopped by application of appropriate corrective action. Repeated exceeding in three consecutive months is considered to be a confirmed negative trend, and corrective action taken by Reliability Control Board is mandated.

Upper control limit is based on statistical calculation of standard deviation covering recent twelve month period. It should not be placed too high because 
in that case negative trends would not be shown and opposite, if it is placed too low, because even small deviations from mean values will trigger exceeding.

Upper control limit is established by multiplying standard deviation above mean value with deviation factor (normally between 2 and 3). Deviation factor is defined by operator and it is depending on dispersion of data - smaller factor is more appropriate for large fleets and greater factor is appropriate for small fleets.

Procedure of UCL establishing is:

a) Calculation of standard deviation:

$$
\sigma=\sqrt{\frac{\Sigma\left(x^{2}\right)-\frac{(\Sigma x)^{2}}{N}}{N-1}},
$$

where: $x$ - monthly value of parameter in observed months; $\sigma$-standard deviation; $N$ - number of observed months for which standard deviation is calculated.

b) Calculation of Upper Control Limit - UCL:

$$
U C L=\bar{x}+\mathrm{k} \sigma,
$$

where: $\bar{x}=\Sigma x / N$,

$k$ - deviation factor (normally between 2 and 3 ).

This calculation of upper control limit (UCL) should be repeated every 12 months. Upper control limit can be increased or decreased by maximum $10 \%$ compared to the previous UCL. Exceptionally, Reliability Control Board can approve a larger change of UCL.

\section{Conclusions}

Application of reliability program is mandatory per current JAR/EASA/FAR regulations for all aircraft operators.

Application of reliability program to a small fleet is highly questionable, due to the fact that small fleets do not generate enough of statistical data to obtain relevant information.

To overcome this problem, it is necessary to:

- Artificially or actually increase the size of the fleet in order to prevent too large dissipation of monthly data,

- establish proper alert values which would not always trigger corrective action based on erratic statistical data generated by a small fleet and still will point out exceeding of ongoing negative trend.

Often, operators maintain reliability programs only to formally satisfy regulatory requirements without real desire to deeply investigate negative trends and take efficient corrective measures.

One of the reasons for such situation is that operators do not understand philosophy of statistical process control. Instead, they concentrate on solving daily, case by case, problems trying to cure symptoms without dealing systematically with problem causes. Reliability program does not react to single events; it discovers system problems and trends that trigger many events. Such problems have a great impact on flight safety and/or economics of an operator.

\section{References}

1. ZERE, I. Appendices to BOEING maintenance engineering evaluations. Seattle, Appendix F - Maintenance Reliability Program, 1995.

2. O'CONNOR, P. D. T. Practical reliability engineering. John Wiley\&Sons, Ltd, West Sussex, England, 2002.

3. ENGA, J. Aircraft inspection and maintenance records. Jeppensen \& Co. GmbH, Germany, 2000.

4. Joint Aviation Regulations JAR OPS-1, JAA. 